\title{
PSMA-PET-CT zum primären Staging von Patienten mit fortgeschrittenem Prostatakarzinom
}

\author{
Cedric Curt Cappel ${ }^{1}$. Denise Dopcke ${ }^{1}$ Jürgen Dunst ${ }^{1}$ \\ Angenommen: 8. Dezember 2020 / Online publiziert: 15. Januar 2021 \\ () Der/die Autor(en) 2021
}

Hintergrund Im Jahr 2016 erhielten 58.780 Menschen in Deutschland die Diagnose eines Prostatakarzinoms, davon befanden sich fast $40 \%$ bereits in den fortgeschrittenen Stadien III und IV nach UICC. Während im Stadium III ohne Vorliegen von regionalen oder Fernmetastasen die tumorspezifische 5-Jahres-Überlebensrate noch bei etwa $100 \%$ liegt, beträgt sie im Stadium IV nur noch 50\% [2]. Als Grundlage für die Therapieplanung werden daher möglichst exakte Staging-Untersuchungen benötigt. Die aktuellen Leitlinien empfehlen bei einem lokal fortgeschrittenen Prostatakarzinom eine CT- bzw. MRT-Untersuchung der Beckenorgane sowie eine Skelettszintigraphie mittels ${ }^{99 \mathrm{~m} T c-}$ basierten Tracern [3]. Eine funktionelle Bildgebung mittels Positronenemissionstomographie (PET) oder PET-CTHybridverfahren ist derzeit nur zur Rezidivdiagnostik nach Anstieg des PSA-Werts trotz kurativer Primärtherapie indiziert. Mit der ${ }^{68} \mathrm{Ga}$-PSMA-PET-CT steht jedoch seit einigen Jahren ein Verfahren zur funktionellen Darstellung von Prostatagewebe zur Verfügung. Als Zielstruktur dient dabei das prostataspezifische Membranantigen PSMA, welches in hohem Maße von Prostatazellen und insbesondere Prostatakarzinomen exprimiert wird [4]. PSMA ist darüber hinaus auch in anderen Drüsengeweben, wie den Speicheldrüsen, sowie in Duodenum, Kolon und Nieren vorhanden, allerdings in weit geringerem Maße [5]. Für die Visualisierung mittels PET dient der synthetische Inhibitor PSMA-11 als Ligand, der einen Komplex mit dem Positronenstrahler ${ }^{68} \mathrm{Ga}$

\section{Originalpublikation Hofman MS et al (2020) Prostate-specific membrane antigen PET-CT in patients with high-risk prostate cancer before curative-intent surgery or radiotherapy (proPSMA). A prospective, randomised, multicentre study. Lancet (London, England) 395(10231).}

cand. med. Denise Dopcke

stu204163@mail.uni-kiel.de

$\triangle$ Prof. Dr. Jürgen Dunst juergen.dunst@uksh.de

1 Klinik für Strahlentherapie Kiel, UKSH, Arnold-Heller-Str. 3, Hs. L, 24105 Kiel, Deutschland bildet [6]. Präklinisch zeigten sich dabei eine gute Kontrastierung von Tumorgewebe [6] sowie eine geringe intraindividuelle Variabilität der Traceraufnahme [7]. In klinischen Studien konnte die PSMA-PET-CT zwar mit einer hohen Sensitivität und Spezifität überzeugen [8], jedoch stammen die bisherigen Ergebnisse größtenteils aus retrospektiven Fallstudien [9]. Um den diagnostischen Nutzen der ${ }^{68} \mathrm{Ga}$-PSMA-PET-CT im primären Staging des Prostatakarzinoms verlässlich und im Vergleich zum bisherigen diagnostischen Standard beurteilen zu können, wurde schließlich die proPSMA-Studie als randomisierte, kontrollierte klinische Studie gestartet [1].

Patientenkollektiv und Methoden Für diese zweiarmige Studie, durchgeführt an zehn Zentren in Australien, wurden zwischen dem 22. März 2017 und dem 2. November 2018 insgesamt 300 Männer rekrutiert und randomisiert einem der beiden Studienarme zugeteilt. Eingeschlossen wurden hierbei lediglich Patienten, welche ein histopathologisch gesichertes Prostatakarzinom und zusätzlich einen PSA Wert $\geq 20 \mathrm{ng} / \mathrm{ml}$, einen ISUP-Grad $\geq 3$ bzw. ein cT3Stadium oder höher aufwiesen. Zudem mussten sie für eine radikale Prostatektomie oder eine Radiotherapie mit kurativer Intention infrage kommen. Die Probanden wurden zufällig im Verhältnis 1:1 auf die beiden Studienarme verteilt: In der Kontrollgruppe erhielten diese ein ,first-line imaging“, bestehend aus einer CT von Becken und Abdomen, sowie eine ${ }^{99 \mathrm{~m}} \mathrm{Tc}-\mathrm{SPECT}-\mathrm{CT}$. In der experimentellen Gruppe bestand das ,first-line imaging“ aus einer ${ }^{68} \mathrm{Ga}-$ PSMA-11-PET-CT unter Nutzung eines standardisierten Protokolls. Im Folgenden wurde bei den Probanden, welche im „first-line imaging“ drei oder mehr uneindeutige Herde mit dem Verdacht auf Fernmetastasen aufwiesen, ein ,cross-over imaging“ mittels der jeweils anderen bildgebenden Methode durchgeführt, um mögliche Nachteile für den Patienten auszugleichen. Im Follow-up nach 6 Monaten wurde die Bildgebung unter Berücksichtigung der ursprünglichen, randomisiert zugeteilten Gruppe wieder- 
holt und wenn nötig erneut um ein „,ross-over imaging“ erweitert.

Als primärer Endpunkt dieser Studie wurde die Genauigkeit des im „first-line imaging“ angewendeten Verfahrens in Bezug auf die Identifizierung pelviner Lymphknotenmetastasen oder Fernmetastasen festgelegt. Diese Genauigkeit wurde mithilfe der "area under the curve“ (AUC) der ROC-Kurve eingeschätzt. Die AUC spiegelt dabei ein Maß für Sensitivität und Spezifität wider. Die Hypothese wurde definiert als eine AUC der PSMA-PET-CT, welche mindestens $10 \%$ größer als die der konventionellen Bildgebungsgruppe sein sollte. Als Referenzstandard, inwiefern ein Befund als positiv hinsichtlich einer pelvinen Lymphknotenmetastase oder Fernmetastase zu werten ist, wurden verschieden vordefinierte Kriterien herangezogen und nach 6 Monaten beurteilt. Harte Kriterien waren die histopathologische Sicherung eines Adenokarzinoms der Prostata und die Veränderung einer Knochenläsion zu einer sklerotischen oder blastischen im „follow-up imaging“. Zusätzlich gab es weitere weiche Kriterien.

Ergebnisse Es konnte herausgestellt werden, dass in der Gruppe der PSMA-PET-CT eine um 27\% absolut höhere AUC und damit höhere Genauigkeit vorlag als in der Gruppe der konventionellen Bildgebung. Sensitivität wie auch Spezifität waren somit deutlich höher unter Anwendung der PSMA-PET-CT. Auch in der Analyse der Subgruppen bestätigte sich dies und zeigte eine um absolut $32 \%$ höhere Genauigkeit bei der Erkennung von pelvinen Lymphknotenmetastasen sowie eine $22 \%$ absolut höhere Genauigkeit bei Fernmetastasen im Vergleich zur konventionellen Bildgebung. Zusätzlich wurden durch die konventionelle Bildgebung mehr uneindeutige Befunde generiert. Dies war in der Kontrollgruppe bei $23 \%$ und in der PSMA-PET-CTGruppe nur bei $7 \%$ der Fall. Außerdem wurde der Einfluss der Bildgebungsart auf mögliche Therapieänderungen untersucht. Bei $28 \%$ mit ,first-line imaging“ mittels PSMA-PET-CT wurde ein mittlerer oder großer Effekt auf den Therapieverlauf im Gegensatz zu 15\% der Patienten in der Kontrollgruppe festgestellt. Auch die Strahlenbelastung der Probanden wurde in dieser Studie betrachtet. Es zeigte sich, dass diese durch das ,first-line imaging“ mit konventioneller Bildgebung, bestehend aus Becken/Abdomen-CT und Szintigraphie, um insgesamt $10,9 \mathrm{mSv}$ höher und somit mehr als doppelt so hoch lag wie mit der PSMA-PET-CT.

Schlussfolgerung der Autoren Die Genauigkeit der PSMAPET-CT übertrifft deutlich die einer CT-Untersuchung bzw. einer Szintigraphie und ermöglicht dabei weniger uneindeutige Befunde. Der Einsatz einer PSMA-PET-CT führt in mehr Fällen zur Änderungen der Behandlungsstrategie.
Auch die Kosten seien im Vergleich zu CT und Szintigraphie günstiger. Die überlegene Genauigkeit der PSMAPET-CT führe zu insgesamt weniger bildgebenden Untersuchungen.

Unter allen betrachteten Gesichtspunkten stellt die PSMA-PET-CT einen geeigneten Ersatz für die konventionelle CT mit Szintigraphie beim Staging von Patienten mit High-risk-Prostatakarzinom und kurativer Therapieintention dar.

\section{Kommentar}

Obwohl PET-CT-basierte Staginguntersuchungen bei vielen Tumorentitäten vielversprechende Studienergebnisse bieten, ist ihre Anwendung im klinischen Alltag noch auf sehr spezielle Indikationen begrenzt. Im Falle des Prostatakarzinoms, der zweithäufigsten Krebserkrankung des Mannes in Deutschland [2], hat die PSMA-PET-CT einzig im Rahmen der Rezidivdiagnostik einen Platz in den aktuellen Leitlinien gefunden. Bisher fehlten aber im Rahmen des primären Stagings bei Patienten mit einem Hochrisikoprofil Daten aus randomisierten, kontrollierten Studien [9]. Daher leistet die proPSMA-Studie einen wertvollen Beitrag für die Einschätzung der PSMA-PET-CT zur Verbesserung des diagnostischen Prozederes. Hofman et al. konnten die diagnostische Überlegenheit der PSMA-PET-CT im Sinne einer deutlich höheren Sensitivität für regionale Lymphknoten- und Fernmetastasen bei mindestens gleichwertiger Spezifität darstellen. Weiterhin lieferte die PSMA-PETCT mehr eindeutige Befunde als das konventionelle, CTbasierte Verfahren und führte bei ca. $28 \%$ der Patienten zur Änderung der geplanten Therapie. Die PSMA-PET-CT hat somit eine unmittelbare therapeutische Konsequenz, nicht nur im Sinne einer Änderung der Therapieintention. Sie ermöglicht im Falle oligometastasierter Erkrankungen auch den Einsatz von bildgesteuerten Hochpräzisionsbestrahlungen (,image-guided radiotherapy“ [IGRT]), welche bereits positive Ergebnisse in klinischen Studien geliefert haben [10].

Die proPSMA-Studie konnte allerdings, bedingt durch das Studiendesign, keinen Effekt auf das Überleben oder die Lebensqualität der Patienten ableiten. Die häufigere Änderung des Therapieregimes mag hierfür ein Indikator sein, jedoch lässt sich dies durch die erhobenen Daten keineswegs belegen. Aufgrund der ohnehin vergleichsweise hohen 5- und 10-Jahres-Überlebensraten bei Prostatakarzinomen sind dringend weitere klinische Studien nötig, um die Langzeiteffekte der verbesserten Diagnostik durch die PSMA-PET-CT abschätzen und eine Aufnahme in die Leitlinien erreichen zu können.

Die hohe Inzidenz des Prostatakarzinoms in Deutschland stellt zudem ein weiteres Problem für den routinemäßigen 
Einsatz der PSMA-PET-CT dar: Bei einer Inzidenz von jährlich etwa 145 Fällen pro 100.000 männlichen Einwohnern fallen statistisch gesehen bundesweit etwa 22.500 Patienten in die Hochrisikogruppen III und IV nach UICC, was die Kapazitäten der PET-Diagnostik bei Weitem übersteigt, zumal die PSMA-PET-CT derzeit nur an spezialisierten Zentren angeboten wird. Hofman et al. spekulieren zwar, dass die Gesamtkosten durch die verbesserte Sensitivität und dadurch geringere Zahl an erforderlichen Kontrolluntersuchungen insgesamt eher sinken würden. Jedoch steht dies den enormen Anschaffungskosten für ein flächendeckendes Angebot an PSMA-PET-CT-Untersuchungen gegenüber. Realistischerweise werden derzeit PSMAPET-CT-Untersuchungen nur in Einzelfällen von den gesetzlichen Krankenkassen bezahlt.

Ein weiterer Schwachpunkt der Studie ist der Vergleich des empfohlenen Verfahrens mit einem CT-basierten Staging, dem die Kontrollgruppe unterzogen wurde. Die deutschen Leitlinien schließen nämlich für das Staging explizit auch MRT-Untersuchungen der Beckenorgane ein, die möglicherweise durch den verbesserten Weichteilkontrast auch eine höhere Sensitivität erreichen könnten. Der entsprechende Vergleich wurde von den Autoren aber nicht adressiert und sollte unbedingt mit weiteren Studien untersucht werden. PET-MRT-Hybridverfahren sind zwar in manchen Zentren bereits etabliert und wurden auch mit dem ${ }^{68}$ Ga-PSMA-11-Tracer in Studien erprobt [11], jedoch liegt ihre Verfügbarkeit noch deutlich unter der einer PETCT. Auch die von Hofman et al. propagierte Verminderung der Strahlendosis durch eine PET-CT-Untersuchung sollte mit Vorsicht aufgenommen werden, weil ein MRT-basiertes Staging mit Sicherheit die einfachere Methode ist zur Reduktion der Strahlendosis.

\section{Fazit}

Die proPSMA-Studie führte bei Patienten mit Prostatakarzinomen einen sorgfältig geplanten und dann auch realisierten Vergleich der PSMA-PET-CT-Technologie mit den herkömmlichen CT-basierten Stagingverfahren durch. Obwohl noch weitere Studien zur Auswirkung auf Überlebensraten und Lebensqualität nötig sind, belegt sie zweifelsfrei die diagnostische Überlegenheit des PET-Verfahrens. Sie leistet damit einen wichtigen Beitrag zu deren langfristiger Etablierung bei der Diagnostik des fortgeschrittenen Prostatakarzinoms und damit zur Optimierung der Patientenversorgung.

\section{Cedric Curt Cappel, Denise Dopcke und Jürgen Dunst, Kiel}

Funding Open Access funding enabled and organized by Projekt DEAL.

Interessenkonflikt C.C. Cappel, D. Dopcke und J. Dunst geben an, dass kein Interessenkonflikt besteht.

Open Access Dieser Artikel wird unter der Creative Commons Namensnennung 4.0 International Lizenz veröffentlicht, welche die Nutzung, Vervielfältigung, Bearbeitung, Verbreitung und Wiedergabe in jeglichem Medium und Format erlaubt, sofern Sie den/die ursprünglichen Autor(en) und die Quelle ordnungsgemäß nennen, einen Link zur Creative Commons Lizenz beifügen und angeben, ob Änderungen vorgenommen wurden.

Die in diesem Artikel enthaltenen Bilder und sonstiges Drittmaterial unterliegen ebenfalls der genannten Creative Commons Lizenz, sofern sich aus der Abbildungslegende nichts anderes ergibt. Sofern das betreffende Material nicht unter der genannten Creative Commons Lizenz steht und die betreffende Handlung nicht nach gesetzlichen Vorschriften erlaubt ist, ist für die oben aufgeführten Weiterverwendungen des Materials die Einwilligung des jeweiligen Rechteinhabers einzuholen.

Weitere Details zur Lizenz entnehmen Sie bitte der Lizenzinformation auf http://creativecommons.org/licenses/by/4.0/deed.de.

\section{Literatur}

1. Hofman MS et al (2020) Prostate-specific membrane antigen PET$\mathrm{CT}$ in patients with high-risk prostate cancer before curative-intent surgery or radiotherapy (proPSMA): a prospective, randomised, multicentre study. Lancet 395(10231):1208-1216. https://doi. org/10.1016/S0140-6736(20)30314-7

2. Robert Koch-Institut, Gesellschaft der epidemiologischen Krebsregister in Deutschland (2019) Krebs in Deutschland für 2015/2016. 12. Ausgabe. RKI, Berlin

3. Leitlinienprogramm Onkologie (Deutsche Krebsgesellschaft, Deutsche Krebshilfe, AWMF) (2018) Interdisziplinäre Leitlinie der Qualität S3 zur Früherkennung, Diagnose und Therapie der verschiedenen Stadien des Prostatakarzinoms, Langversion 5.0, AWMF Registernummer: 043/022OL. http://www.leitlinien programm-onkolo-gie.de/leitlinien/prostatakarzinom/. Zugegriffen: 24. Okt. 2020

4. Sweat SD et al (1998) Prostate-specific membrane antigen expression is greatest in prostate adenocarcinoma and lymph node metastases. Urology 52(4):637-640

5. Silver DA et al (1997) Prostate-specific membrane antigen expression in normal and malignant human tissues. Clin Cancer Res 3(1):81-85

6. Afshar-Oromieh A et al (2013) PET imaging with a 68[Ga]galliumlabelled PSMA ligand for the diagnosis of prostate cancer: biodistribution in humans and first evaluation of tumour lesions. Eur J Nucl Med Mol Imaging 40(4):486-495

7. Olde Heuvel $J$ et al (2020) Day-to-day variability of $\left[{ }^{68} \mathrm{Ga}\right] \mathrm{Ga}-$ PSMA-11 accumulation in primary prostate cancer: effects on tracer uptake and visual interpretation. EJNMMI Res 10:132

8. Maurer T et al (2016) Diagnostic efficacy of (68)gallium-PSMA positron emission tomography compared to conventional imaging for lymph node staging of 130 consecutive patients with intermediate to high risk prostate cancer. J Urol 195(5):1436-1443

9. Perera M et al (2016) Sensitivity, specificity, and predictors of positive ${ }^{68} \mathrm{Ga}$-prostate-specific membrane antigen positron emission tomography in advanced prostate cancer: a systematic review and meta-analysis. Eur Urol 70(6):926-937 
10. Marzec J et al (2020) ${ }^{68} \mathrm{Ga}$-PSMA-PET/CT-directed IGRT/SBRT for oligometastases of recurrent prostate cancer after initial surgery. Acta Oncol 59(2):149-156

11. Grubmüller B et al (2018) PSMA ligand PET/MRI for primary prostate cancer: staging performance and clinical impact. Clin Cancer Res 24(24):6300-6307 